\title{
The Effect of General Relativity and Equation of State on the Adiabatic Collapse and Explosion of a Stellar core.
}

N. Sack aind I. Lichtenstadt

Racah Institiute of Physics

The Hebrew University of Jerusalem, ISRAEL 91904

The collapse of the iron core of massive stars $\left(M \geq 8 M_{0}\right)$ is initiated by photodissociation and electron capture. The collapse of the inner core proceeds homologously until it is stopped by the stiffness of the equation of state (hereafter EOS) at nuclear density and it stops or rebounds. A shock forms at the edge of homology. The initial strength of the shock increases with the velocity difference between the inner and outer cores, i.e. It increases with a larger rebound of the inner core. The uniterrupted propagation of this prompt shock through the remainder of the core to the stellar mantle, where it can deliver enough energy to blow of the loosely bound outer layers, has long been proposed as the mechanism of type II supernovae explosions. However most authors did not get an explosion as a result of the prompt mechanism. Recently Baron et al. (1985) reported that the combination of General Relativity (GR) with a relatively soft EOS at nuclear densities leads to a much greater blow of $f$ than they got with Newtonian hydrodynamics. In order to see where purely hydrodynamical effects are important, namely for what EOS the GR outburst is greater than the Newtonian, we did a set of pure hydrodynamical adiabatic calculations (complete neutrino trapping) with different EOS above nuclear densities, turning the GR terms on and of Neutrino leakage, which we do not incorporate, usually leads to harmful energy losses.

We used a $1.35 \mathrm{M}_{0}$ iron coreformed from a $12 \mathrm{M}_{\mathrm{o}}$ star calculated by Woosley et al. (1984), and the compressible liquid drop model EOS of Lamb et al. (1978) up to nuclear densities. At higher densities we added to the lepton pressure a cold pressure as given by Baron et al. in the

following form: $\quad P_{\text {cold }}=\frac{K_{0} \cdot \rho_{0}}{9 \cdot \tau}\left[\left[\frac{\rho}{\rho_{0}}\right]^{\gamma}-1\right]$

where $K_{0}=9 \cdot(\mathrm{dP} / \mathrm{d} \rho)_{\rho=\rho}$ is the nuclear incompressiblity at saturation, $\rho_{0}$ is the nuclear density and $\gamma$ is the high density adiabatic index. Thus we have a two parameter ( $K_{0}$ and $r$ ) form for the cold nuclear EOS. We used three different values for $K_{0}: 125,200$ and $275 \mathrm{MeV}$, where $\mathrm{K}_{0}=200 \mathrm{MeV}$ is a conservative value (Blaizot 1980) and the other two 
represent a distinct change to the low and high incompressibilities. For $r$ we used the values $2,2.5$ and 3 . All calculations were done twice. turning GR terms of $f$ and on. The results are represented in the following table:

\begin{tabular}{|c|c|c|c|c|c|c|c|}
\hline $\mathrm{K}_{\mathrm{o}}(\mathrm{MeV})$ & $\boldsymbol{\gamma}$ & $\mid \begin{array}{c}\rho_{\mathrm{B}}^{\mathrm{NR}} / 10^{14} \\
\mathrm{~g} \cdot \mathrm{cm}^{-3}\end{array}$ & $M_{e j}^{N R} / M_{o}$ & $\mid \begin{array}{l}\mathrm{E}_{\mathrm{ej} / 10^{50}}^{\mathrm{NR}} \\
(\mathbf{e r g})\end{array}$ & $\rho_{\mathrm{B}}^{\mathrm{GR}} / \rho_{\mathrm{B}}^{\mathrm{NR}}$ & $\mathrm{M}_{\mathrm{ej}}^{\mathrm{GR}} / \mathrm{M}_{\mathrm{ej}}^{\mathrm{NR}}$ & $\mid E_{e j}^{G R} / E_{e j}^{N R}$ \\
\hline 125 & 2.0 & 7.7 & 0.093 & 2.2 & - & - & - \\
\hline 125 & 2.5 & 6.6 & 0.093 & 3.8 & 2.6 & 1.1 & 0.66 \\
\hline 125 & 3.0 & 5.9 & 0.093 & 3.6 & 1.8 & 0.01 & 0.036 \\
\hline 200 & 2.0 & 5.7 & 0.093 & 2.6 & 3.1 & 1.4 & 0.77 \\
\hline 200 & 2.5 & 5.2 & 0.093 & 2.5 & 1.9 & 0.04 & 0.03 \\
\hline 200 & 3.0 & 4.9 & 0.083 & 2.5 & 1.6 & 0 & 0 \\
\hline 275 & 2.0 & 4.9 & 0.083 & 4.7 & 2.2 & 0.03 & 0.13 \\
\hline 275 & 2.5 & 4.6 & 0.083 & 4.7 & 1.7 & 0 & 0 \\
\hline 275 & 3.0 & 4.4 & 0.083 & 2.9 & 1.5 & 0 & 0 \\
\hline
\end{tabular}

Superscripts NR and GR denote the Newtonian and GR results respectively. $p_{B}$ is the maximum density at bounce, $M_{e j}$ is the ejected mass and $E_{e j}$ is the total energy associated with it.

In the Newtonian calculations, we see the dependency of the bounce density $\rho_{B}$ on $K_{0}$ and $\gamma$. Softer nuclear cold EOS results in a deeper penetration. The ejected mass is almost independent of $K_{0}$ and $r$. It depends mostly on the size of the homologous core, which does not change here. The size of the homologous core depends on the effective adiabatic index $\Gamma_{\text {eff }}$ during the collapse and not on $K_{0}$ and $r$ and is about $0.95 \mathrm{M}_{0}$. The prompt shock always originates at the edge of the homologous core moving into the looslier bound mantle, and the mass bifurcation point does not change much with $K_{0}$ and $r$. On the other hand the energy of the ejecta, does depend on the details of penetration at bounce. General relativity changes the Newtonian picture : as expected,

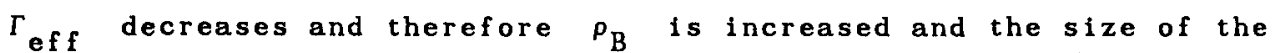
homologous core decreases to about $0.6 \mathrm{M}_{\mathrm{o}}$. For $\mathrm{K}_{0}=125 \mathrm{MeV}$ and $r=2$ the collapse procceeds to a black hole, so that we do not represent GR values. The high incompressibility $K_{0}=275 \mathrm{MeV}$, in GR supresses the ejected mass for all $\gamma$ used. For the intermediate $K_{0}=200 \mathrm{MeV}$, only $r=2$ benefits from GR and for the low $K_{0}=125 \mathrm{MeV}$ we need $r=2.5$ to get an ejecta. All the other cases resulted in negative or marginal explosions. Still even in the cases of the two GR successful explosions the ejected mass is greater for GR while the ejected energy is reduced by GR effects. 
As Baron et al. pointed out there is a constraint on the EOS from the observations of neutron-star gravitational masses to be about 1.5 $M_{0}$. There is a maximum for the neutron-star mass for a given EOS and this value increases monotonically with the stifness of the EOS. They summarized their results in the following formula:

$$
M_{\max }=1.07 \cdot(\gamma-1) \cdot\left(K_{0} / 230\right)^{1 /(2 \gamma-2)} M_{0}
$$

Using this formula we obtained the minimal $r\left(r^{m i n}\right)$ necessary to get a $1.5 \mathrm{M}_{0}$ neutron star for the different incompressibilities we used. The minimal $r$ values are 2.7, 2.5 and 2.3 for $K_{o}=125.200$ and $275 \mathrm{MeV}$ respectively. These minimal adiabatic indices are larger than the values we find necessary to produce energetic explosions, suggesting that the relativistic amplification of the rebound amplitude may not be the mechanism responsible for supernova explosions.

The differences between our results and those of Baron et al. might be due the different EOS, the usage of a leakage scheme and due to their dependency of $\mathrm{K}_{\mathrm{o}}$ on $\mathrm{Y}_{\mathbf{e}}$.

\section{CONCLUSIONS}

1. GR effects are important in the collapse and explosion of an iron stellar core. Even when the net ejected masstenergy is similar, the inner hydrodynamics is different, mainly by forming a smaller homologous core. We found that the extra energy gained by the deeper bounce penetration is more than wasted on heating up the thicker mantle. 2. There is a narrow regime near the points: ( $\left.K_{0}=125 \mathrm{MeV}, \gamma=2.5\right)$ and $\left(K_{0}=200 \mathrm{MeV}, r=2\right)$ that permits a successful explosion. However, as Baron et al. themselves realize, these values for the EOS parameters are ruled out by the masses observed for cold neutron stars, if the EOS of neutron-star matter is not stiffer than our EOS.

3. Allowing neutrino leakage would allow some small reduction in $K_{0}$. but would lead to neutrino energy losses, larger in the GR case than in the Newtonian, because of the smaller inner core and the larger hot overlaying mantle.

\section{References:}

Baron, E. Cooperstein, J., and Kahana, S. 1985, Phys. Rev. Letters, 55, 126 .

Blaizot, J.P. 1980, Phys. Rep.,64, 171 .

Lamb, D. Q., Lattimer, J.M., Pethick, C., and Ravenhall, D.G. 1978 , Phys. Rev. Letters, 41, 1623.

Woosley, S. E., and Weaver, T. A. 1984, Bull. Am. Astron. Soc., 16, 971. 